This item was submitted to Loughborough's Research Repository by the author.

Items in Figshare are protected by copyright, with all rights reserved, unless otherwise indicated.

\title{
SmartARM: A smartphone-based group activity recognition and monitoring scheme for military applications
}

PLEASE CITE THE PUBLISHED VERSION

https://doi.org/10.1109/ANTS.2017.8384149

PUBLISHER

IEEE

VERSION

AM (Accepted Manuscript)

\section{PUBLISHER STATEMENT}

This work is made available according to the conditions of the Creative Commons Attribution-NonCommercialNoDerivatives 4.0 International (CC BY-NC-ND 4.0) licence. Full details of this licence are available at: https://creativecommons.org/licenses/by-nc-nd/4.0/

\section{LICENCE}

CC BY-NC-ND 4.0

\section{REPOSITORY RECORD}

Mukherjee, A., Sudip Misra, P. Mangrulkar, Muttukrishnan Rajarajan, and Yogachandran Rahulamathavan. 2019. "Smartarm: A Smartphone-based Group Activity Recognition and Monitoring Scheme for Military Applications”. figshare. https://hdl.handle.net/2134/36323. 


\title{
SmartARM: A Smartphone-based Group Activity Recognition and Monitoring Scheme for Military Applications
}

\author{
A. Mukherjee ${ }^{1}$, Sudip Misra ${ }^{1}$, P. Mangrulkar ${ }^{1}$, Muttukrishnan Rajarajan ${ }^{2}$, and Yogachandran Rahulamathavan ${ }^{3}$ \\ ${ }^{1}$ Department of Computer Science and Engineering, Indian Institute of Technology Kharagpur, India \\ Email: anandarupmukherjee@ieee.org \\ ${ }^{2}$ School of Mathematics, Computer Science. \& Engineering, City University London, United Kingdom \\ Email: r.muttukrishnan@city.ac.uk \\ ${ }^{3}$ Institute for Digital Technologies, Loughborough University London, United Kingdom \\ Email: Y.Rahulamathavan@1boro.ac.uk
}

\begin{abstract}
In this paper we propose SmartARM - a Smartphone-based group Activity Recognition and Monitoring (ARM) scheme, which is capable of recognizing and centrally monitoring coordinated group and individual group member activities of soldiers in the context of military excercises. In this implementation, we specifically consider military operations, where the group members perform similar motions or manoeuvres on a mission. Additionally, remote administrators at the command center receive data from the smartphones on a central server, enabling them to visualize and monitor the overall status of soldiers in situations such as battlefields, urban operations and during soldier's physical training. This work establishes - (a) the optimum position of smartphone placement on a soldier, (b) the optimum classifier to use from a given set of options, and (c) the minimum sensors or sensor combinations to use for reliable detection of physical activities, while reducing the data-load on the network. The activity recognition modules using the selected classifiers are trained on available data-sets using a testtrain-validation split approach. The trained models are used for recognizing activities from live smartphone data. The proposed activity detection method puts forth an accuracy of $80 \%$ for real-time data.

Index Terms-Smartphone, Activity Recognition, Sensors,
\end{abstract} Classifier, Group Activity Learning

\section{INTRODUCTION}

The use of smartphones in military applications although limited, but is seeing a gradual rise in acceptance due to its high computation capability and compactness of size [1]. As the communication channels are encrypted and communication stations in battlefield are manned by manual and automatic defense mechanisms, the communicating infrastructure is considered to be un-corruptable in our selected scenario. Our proposed SmartARM system as shown in Fig. 1 , is an endeavor to develop a real-time smartphone-based soldier localization [2] and monitoring system enabling the military planners and commanders to acquire situational awareness of a battlefield from the activity status of the participating soldiers, instead of relying only on verbal reports. The activity of a soldier or his/her group remotely provides health-stress conditions byfi tting it on a pre-trained activity recognition model indicating normal or abnormal activity of a soldier with respect to his group. In case, all the soldiers in a region are engaged in similar activity, the groupbased activity recognition system infers the group's activity - running, jumping, crouching, lying, and others. On the contrary, if some of the soldiers show significant deviation from the group's detected activity, the activity detection systemfl ags it as an abnormality, upon which the commander is alerted. This system is similar to Ambient Assisted Living (AAL) architectures, such as the ones proposed by Lloret et al. [3] and Ullah et al. [4]. However, our approach depends on manually carried activity sensing platforms - smartphones instead of inferring emergency situations from communication channel message exchanges. Additional challenges of a soldier monitoring system include sensor placement on the soldier to achieve maximum accuracy of activity detection without hindering the soldier's movements, unlike standard approaches such as the one demonstrated by Lemmens et al. [5].

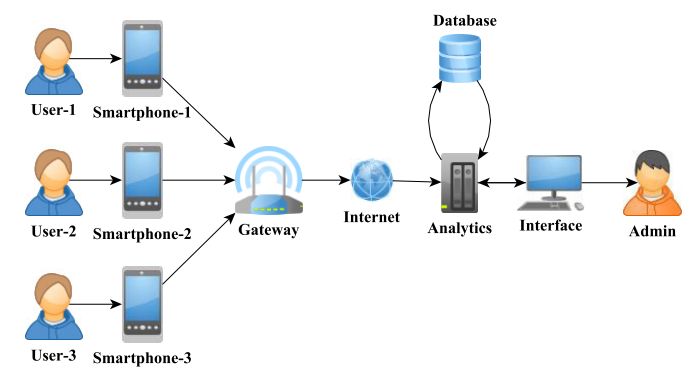

Fig. 1. The SmartARM architecture showing the network set-up and dataflow to a remote server over the Internet.

The following smartphone sensors are used for SmartARM - Accelerometer $(\alpha)$, Magnetometer $(\mu)$ and Gyroscope $(\gamma)$ [5]. The raw accelerometer values $\alpha_{d}(x, y, z)$, along $x, y$ and 
$z$ axes of the smartphone, for a sensed force $(F(x, y, z))$ acting on a mass $(m)$ is $( \pm g)-\frac{\sum F(x, y, z)}{m}$, where, $g \simeq 9.8 \mathrm{~m} / \mathrm{s}^{2}$ is the acceleration due to gravity. $\alpha_{d}(x, y, z)$ is translated to linear acceleration by passing it through a high passfilter (HPF) $\left(\mathcal{H}_{\omega}\right)$ to generate the linear acceleration, as shown in Equation 1.

$$
\alpha=\left[\alpha_{x}, \alpha_{y}, \alpha_{z}\right]=\mathcal{H}_{\omega}\left(\alpha_{d}(x, y, z)\right)=( \pm 2 g)-\frac{\sum F(x, y, z)}{m}
$$

The $\pm 2 g$ term is present as a result of sensitivity adjustments of Inertial Measurement Unit (IMU) sensor of the smartphone, which provides sensitivities in the range of $\pm\left. 2^{x} g\right|_{x=1,2,3,4}$. This value is determined by the respective sensor's datasheet. Similarly, the raw magnetometer and gyroscope values of the smartphone are represented by $\left(\mu_{x}, \mu_{y}, \mu_{z}\right)$ and $\left(G_{x}, G_{y}, G_{z}\right)$, respectively. For gyroscopes, these are modified to get thefi nal value $\left(\gamma \supset\left(\gamma_{x}, \gamma_{y}, \gamma_{z}\right)\right)$ according to Equation 2.

$$
\gamma=\left[\frac{G_{x}}{\sqrt{G_{x}^{2}+G_{y}^{2}+G_{z}^{2}}}, \frac{G_{y}}{\sqrt{G_{x}^{2}+G_{y}^{2}+G_{z}^{2}}}, \frac{G_{z}}{\sqrt{G_{x}^{2}+G_{y}^{2}+G_{z}^{2}}}\right]
$$

$\alpha, \mu$ and $\gamma$ are transmitted over the network to the remote server. According to Algorithm 1, the incoming data to the server, $\mathcal{D}$ is of the form $\left[\alpha_{x}, \alpha_{y}, \alpha_{z}, \mu_{x}, \mu_{y}, \mu_{z}, \gamma_{x}, \gamma_{y}, \gamma_{z}\right]$. This algorithm generates output in the form of probability scores of classes $P\left(C_{k}\right)$ for each individual user equipped with the SmartARM smartphone. Multiple $(n)$ individual activity reports $\left(P_{n}\left(C_{k}\right)\right)$ are combined to generate an ensemble $\left(G_{e}\right)$ for a certain GPS-grid $\left(G P S_{x}\right)$, such that $\left[P_{1}\left(C_{k}\right), P_{2}\left(C_{k}\right), \cdots, P_{n}\left(C_{k}\right)\right], \forall P_{n}\left(C_{k}\right) \in G P S_{x}$. The primary assumptions for implementing this system are enumerated in Assumptions 1 and 2.

Assumption 1: The smartphones and the server are connected by a network. The smartphone uploads data to the server at a user-configurable rate (here, $t=200$ samples per second). For faster operation and energy savings, the sampling rate can be lowered. However, it is not advisable to lower it below $t=50$ samples per second, as it tends to miss relevant information from the recorded data.

Assumption 2: The group objective or mission is known to the individual group members as well as the central missioncontrol. The group and individual status are inferred based on the pre-defined mission.

\section{A. Motivation}

Soldiers in a battlefield depend on highly coordinated movements and actions of their teams. Sudden and jerky movements during evasive or attacking actions in battlefields are common but, may force wrong detection of activities by an activity detection system such as the one described by Anjum et al. [6]. Additionally, a soldier monitoring system demands energy efficieny to endure long missions, as well as, compactness of form, so as not to hinder the soldier's natural movements.

\section{B. Contributions}

In this work, a smartphone-based activity recognition scheme is evaluated against various placement positions of the smartphone on the human body. This work additionally proposes a scheme for smartphone-based monitoring of group activities, generally associated with military units. In lieu of these tasks, the following contributions have been made in this work:

1) Placement of data acquisition platform on the soldier in such a manner that it does not hinder his/her natural movements.

2) Selection of the sensor or least number of sensor combinations instead of using all available sensors to minimize data transmission load on a constrained transmission network.

3) A group-based validation of activities detected for soldiers in battlefields to reduce chances of mis-interpreted activities where a subject is exposed to sudden and abrupt motion, making individual-based activity detection approaches highly errorenous.

\section{RELATED WORKS}

Positional sensor embedded smartphones are being increasingly used for human activity recognition and monitoring due to their intrusive presence in the society, and considerable computing power. Wang et al. [7] used the smartphone built-in accelerometer and gyroscope sensors to recognize simultaneous, as well as separate human physical activities. Their feature selection method, and online activity recognizer scheme reports better activity generalization ability and improved power savings in the smartphone. Similarly, Chen et al. [8] proposed a performance analysis method for motion-sensor behavior in smartphone-based human activity sequences. Their work utilized data segmentation using their cycle detection algorithm, and data characterization using time, frequency and wavelet domain operations, which resulted in an F-score of $96.26 \%$. Segundo et al. [9] proposed a human activity classification scheme which employs Hidden Markov Models (HMMs) applied on publicly available smartphone-based human activity datasets. Their approach reported an activity recognition error rate of $2.5 \%$. Taufeeq Uddin et al. [10] proposed importance score driven random forests to recognize activities and postural transitions on smartphones to reduce fall in recognition accuracies due to these transitions. Their methods achieved 100\% detection accuracies during human activity recognition on benchmark datasets.

One of the predominantly used applications of human activity recognition is in the healthcare sector. Actitracker, a smartphone-based activity recognition method developed by Weiss et al. [11] doubles as a health assistrant. Their system allowed people to set personal activity goals and monitor their progress toward these goals. Another one of the diverse usages of human activity recognition can be found in the work by Lee and Song [12]. They proposed a machine 
learning system operational on a smartwatch and a smartphone to recognize stereotyped movements in children with developmental disabilities. Their system achieved an average recognition accuracy of $91 \%$ on individual training datasets. Similarly, Grunerbl et al. [13] proposed a smartphone-based system which can recognize depressive and manic states and detect state changes of patients suffering from bipolar disorder. They reported accuracies of $76 \%$ by fusing sensor modalities and state change detection precision and recall of over $97 \%$. Smartphone-based human activity sensing can be found even in domains such as elderly care, as proposed by Cardoso et al. [14].

Synthesis: The various works in the domain of smartphone-based human activity recognition for various applications are mainly targeted for individual users in a predominantly civillian populace. As very selective domains can incorporate group activities for garnering collective information, works on group activity recognition and validation systems are considerably lacking. Additionally, none of these works address the problem of missing or corrupted smartphone data, during transmission.

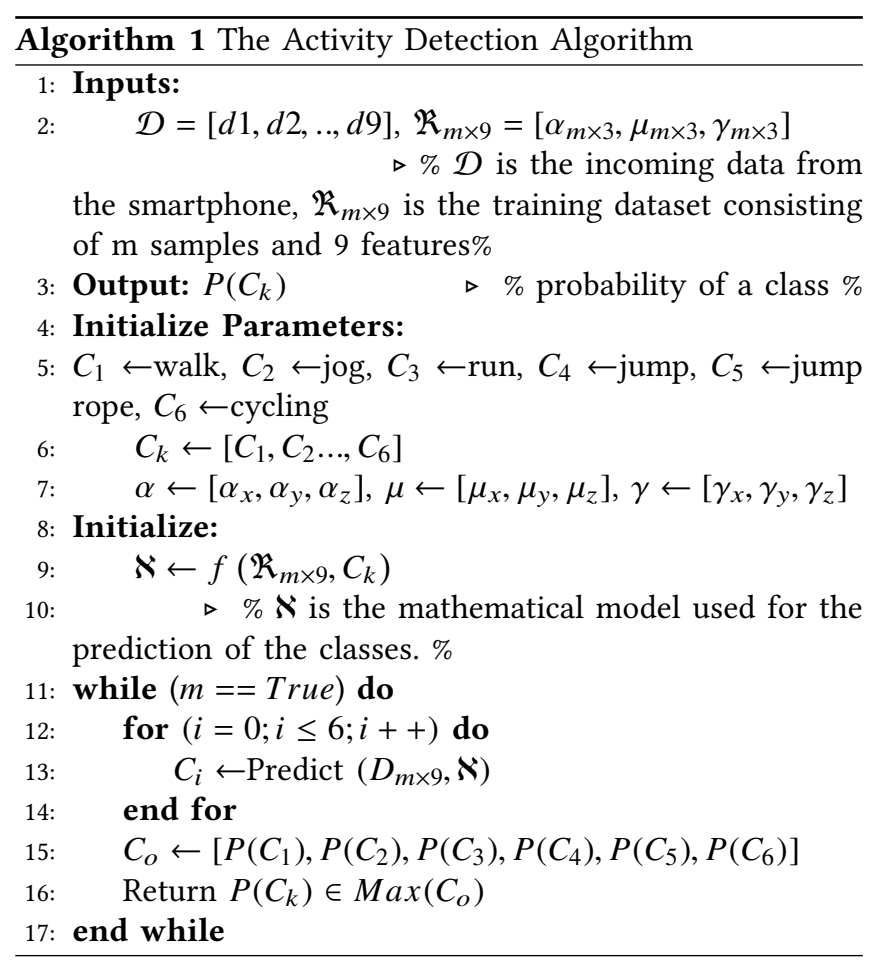

\section{SystemDescription}

The SmartARM architecture consists of 2 main parts - (a) individual smartphone-side data generation, and (b) serverside analysis. The sensor data $\mathcal{D}$ from each member $\mathcal{K}_{i}$ of the group $\mathcal{M}$ is sent continuously to the server using the Internet or locally available WLAN infrastructure. The server has provision for parallel storage and analysis of the incoming data-logs. The generated probability of activity $P\left(C_{K}\right)$, obtained byfi tting the incoming data to pre-trained model, from Algorithm 1 is applied on Algorithm 2 to infer group status as well as individual status with respect to the group. Functions Temporal Ensemble, Group Ensemble and Individual Status supplement Algorithm 2.

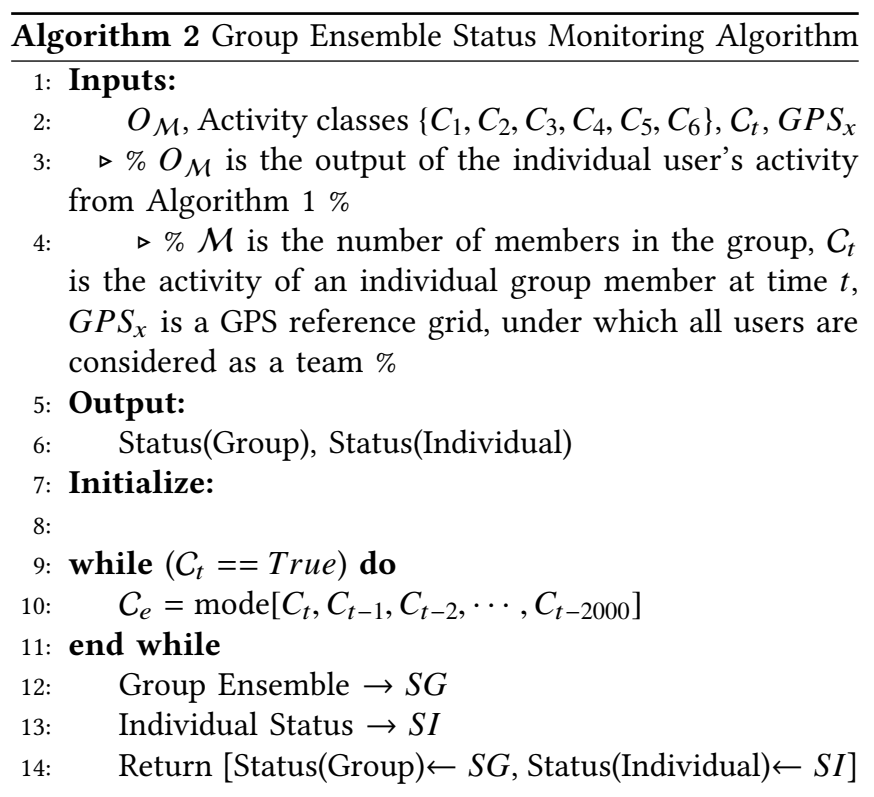

\section{A. Data}

A sanitized training data-set is used for generating the activity recognition model, as outlined in Algorithm 1. The data-set used is REALDISP Activity Recognition Dataset [15], available at the UCI Machine Learning Repository [16]. This data-set consists of 33 activities, recorded using 9 sensors, placed on 17 subjects in 3 different scenarios. Out of these 33 activities, we choose 6 - walk, jog, run, jump, jumprope and cycle - for all 17 subjects in a single scenario. Additionally, Live-data is recorded on the same six activities for approximately 18 minutes, wherein 4 minutes each are assigned for continuous walking, jogging and running, followed by a minute each of jumping and jump-rope and finally, 4 minutes of cycling. The live-data is meant only for checking the effectiveness of the proposed method on actual scenarios, and hence, the time taken for each activity is noninfluential. This data is uploaded to the remote server and fit onto pre-trained activity recognition modules, as outlined in Algorithm 1. The data is uploaded for a group-size of 6 people, which is the most widely used size of a military special operations teams world-wide. However, this approach can be scaled-up to include larger groups/teams working in tandem.

\section{B. Experimental Setup}

We use two different Android-enabled smartphones in this work - Samsung Galaxy S Duos and Xiaomi Mi 4i. Five positions on users are identified for placing the smartphone, so that the placed smartphone does not in any way affect the physical activities of the individual user. The positions 


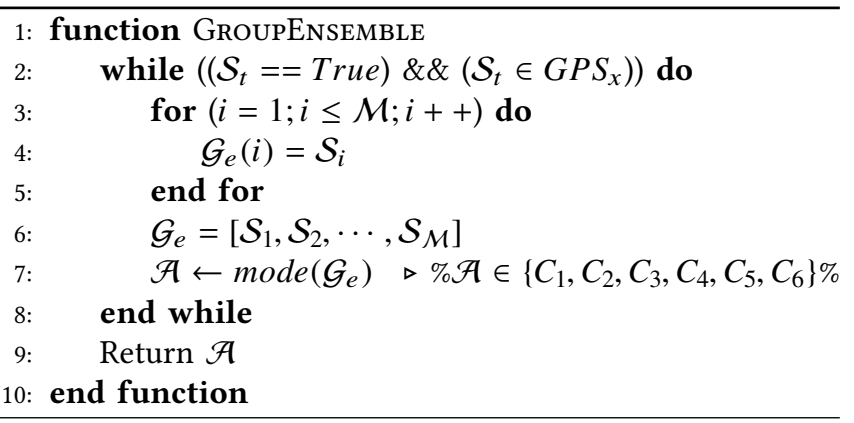

selected are - Right Upper Arm (RUA), Left Upper Arm (LUA), Back, Left Thigh (LT), and Right Thigh (RT), as shown in Fig. 2. Based on established works of relatively similar essence [6],fi ve classifiers are chosen for classifying the incoming smartphone signals - Decision Trees (A), Naïve Bayes (Gaussian) classifier (B), K-Nearest Neighbor(KNN) (C), Support Vector Machines (SVM) (D) and Random Forest (E) - which are trained on the REALDISP dataset [16].

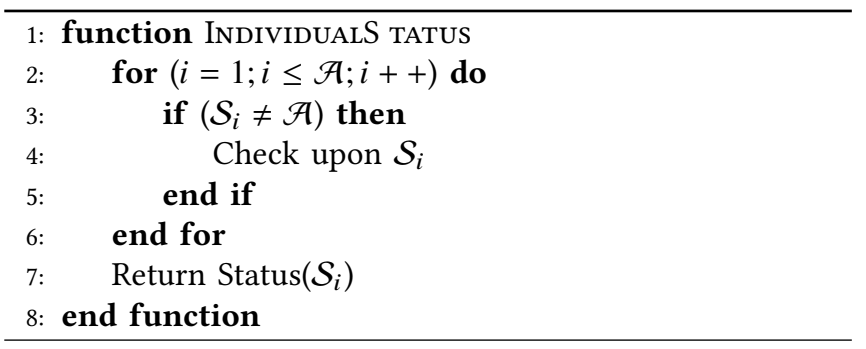

These classifiers are behaviourally contrasting, which provides for a relativistic understanding of each method in classifying the data in a challenging scenario such as the one chosen by us. Decision Trees operate on the basis of certain user-defined decision-rules which convert a problem into a tree-like structure to decide the probability of a data-point belonging to a class. Similar to Decision Trees, Random Forests use an ensemble of all the outputs generated from multiple Decision Trees to arrive at thefi nal probability of the data-point belonging to each class. A Naïve Bayes classifier calculates the normalized mean and standard deviation of the data to assign the probability of belongingness of the datapoint for each class. A KNN classifier is non-parametric and arrives at the data-point'sfi nal class belonging probability by taking the majority vote on the frequency of occurrence the data-point's K-nearest neighbours. Finally, an SVM is a non-probabilistic, binary, linear-classifier which uses a Radial Basis Function kernel (in our case) with "one-versus-all" mapping to convert the input data into linearly separable classes. The server-side algorithms for utilizing the data, classifiers and data-sets for inferring activities are outlined in Algorithms 1 and 2. Various challenges are addressed with respect to the activities of an individual user such as - the optimal sensor placement position on the subject without hindering his physical movements, sensor or specific sensor combinations for generating maximum activity-classification accuracy from minimum sensor data and counteracting the effects of missing or noise-corrupted sensor data at the server.

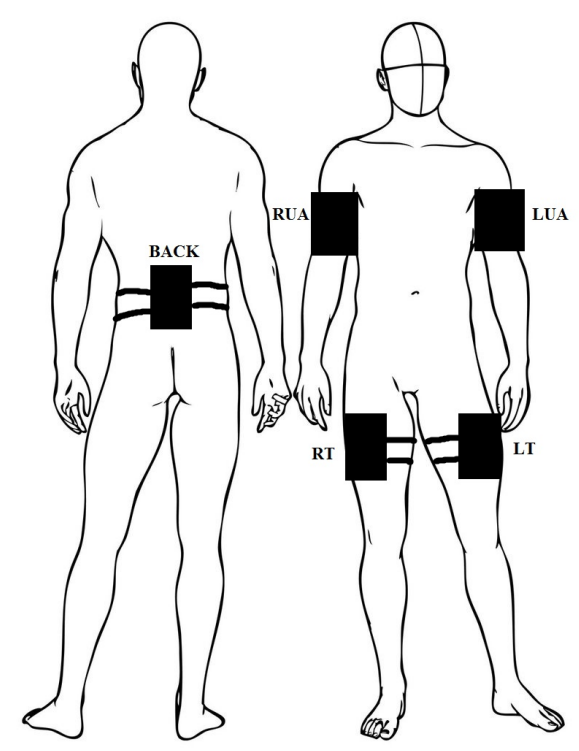

Fig. 2. The relative position of the smartphone on the human body.

\section{Results}

This section discusses the results obtained for the setup discussed in Section III-B, and helps narrow-down the sensor placement position, sensor or their combinations and the suitable classifier for classifying various activities. Figs. 3 and 4 summarize the respective results offi nding the optimal position for sensor placement and effect of various sensors and their combination on the accuracy of activity identification using various classifiers. Additionally, eachfi gure shows the performance of these different classifiers in accurately classifying each activity from the REALDISP dataset [16]. A train-test-validation approach has been followed in analyzing the performance of the classifiers in the activity detection tasks. The data-set is split into train-test-validation sets in the ratio of $50: 30: 20$. In addition to following a train-testvalidation split based approach, the trained classifier models are also used for activity detection on live-data, which is manually validated for detected activities. Fig. 5 shows the performance of the chosen classifiers in correctly recognizing the Live data activity on the REALDISP dataset-based models, which are pre-trained in the server according to Algorithms 1 and 2. There exists corruption of data during sensor-noise or improper decoding of data at the server-end, resulting in errorenously formatted data which is counteracted by the use of an imputing function to weed out these erroroneous instances.

\section{A. Sensor Placement Position}

The comparative performance of various smartphone placement positions with respect to a soldier in action is 
TABLE I

Placement position BASEd MOdel training Metrics for RFClAsSifier USING ALL THREE SENSORS. THE VALUES LIE BETWEEN 0 AND1.

\begin{tabular}{lllll}
\hline Pos & Precision & Recall & f1-score & Accuracy \\
\hline \hline RUA & 0.97 & 0.97 & 0.97 & 0.9651 \\
LUA & 0.97 & 0.97 & 0.97 & 0.9712 \\
BACK & 0.96 & 0.96 & 0.96 & 0.9604 \\
LT & 0.96 & 0.96 & 0.96 & 0.9559 \\
RT & 0.96 & 0.96 & 0.96 & 0.9590 \\
\hline
\end{tabular}

discussed in this section. The comparison between the selected classifiers and sensor placement positions is shown in Fig. 3, which is generated by using all three sensors $(A+G+M)$. The LUA position of the smartphone repeatedly provides best classification accuracy for all the chosen classifiers, except for the Naïve Bayes classifier, where the Back position provides the highest accuracy of classification. The highest accuracy of classification achieved using LUA as the smartphone position is for the Random Forest (RF) classifier at 97.12\%. For a speedier classification, the K-Nearest Neighbour (KNN) classifier can be chosen, which generates an accuracy of $96.07 \%$ and is the fastest among the chosen classifiers. The classification metrics are shown in Tables I and II, which signify the absence of opportunistic performance, such as over-fitting of data. Table I shows the performance of the best classifier - Random Forest - in detecting activities for various sensor placement positions on the soldier. Similarly, Table II shows the performance metrics for the best adjudged sensor placement position - LUA (as shown in Fig. 3) taking the readings from all three sensors together.

TABLE II

MODEL TRAINING METRICS FOR THE CHOSEN CLASSIFIERS USING LUA AS THE SENSOR PLACEMENT POSITION WITH THE USE OF ALL THREE SENSORS. THE VALUES LIE BETWEEN 0 AND1.

\begin{tabular}{lllll}
\hline Classifier & Precision & Recall & f1-score & Accu. \\
\hline \hline Random Forest & 0.97 & 0.97 & 0.97 & 0.9712 \\
KNN & 0.96 & 0.96 & 0.96 & 0.9607 \\
SVM & 0.07 & 0.27 & 0.11 & 0.2694 \\
Naive Bayes & 0.77 & 0.76 & 0.76 & 0.7647 \\
Decision Trees & 0.93 & 0.93 & 0.93 & 0.9324 \\
\hline
\end{tabular}

\section{B. Sensor(s) Selection}

Having evaluated the best classifier amongst the chosen ones and the optimum sensor placement position on the soldier using all three sensors $(A+G+M)$ together, we now aim to reduce the data-load on the remote server by evaluating the performance of individual sensors or sensor combinations in activity detection to generate results comparable to the use $A+G+M$. Fig. 4 shows the performance of various classifiers in classifying the activities for standalone smartphone sensors, as well as their combination - Accelerometer and Magnetometer (AM), Accelerometer and Gyroscope (AG), Magnetometer and Gyroscope (MG). The RF classifier



Fig. 3. Performance of classifiers in detecting activities with respect to varying smartphone positions on the human body.

consistently yields the best classification accuracy for all the three individual sensors - accelerometer, magnetometer, gyroscope - at $75.41 \%, 87.87 \%$, and $60.68 \%$, respectively. In the combination mode, the RF classifier again provides consistent performance and highest classification accuracy at $89.91 \%$ for $\mathrm{AG}, 95 \%$ for $\mathrm{AM}$ and $94.7 \%$ for MG. As the incoming data for a combination of sensors results in larger data load on the server, the KNN can be considered for a faster performance as compared to the other classifiers, as is evident in Fig. 4.

\section{Counteracting Missing Sensor Values}

Fig. 5 summarizes the performance of the chosen classifiers in classifying data with sudden missing or noise-corrupted sensor values in live incoming data. A mode-based imputing function $\left(\mathcal{I}\left(d_{i}\right)\right)$ is used tofi $1 \mathrm{l}$ in the missing or corrupted sensor values prior tofi tting on the pre-trained model in the server for the sake of maintaining continuity of data. $\mathcal{I}\left(d_{i}\right)$ is calculated as, $\mathcal{I}\left(d_{i}\right)=\operatorname{mode}\left\{\left\{\left[d_{i}(t)\right\}\right]_{t=(i-1)}^{(i-11)}\right\}$. Both the $\mathrm{RF}$ classifier and $\mathrm{KNN}$ demonstrate an activity classification accuracy of over $85 \%$ in conjunction with $\mathcal{I}\left(d_{i}\right)$. The RF classifier performs marginally better than KNN with an accuracy of $87 \%$ however, the RF classifier additionally acts as a feature extractor therefore, eliminating the need for separate feature extraction techniques such as Dynamic Time Warping (DTW).

\section{Conclusion}

The SmartARM system provides a comprehensive on-field soldier status monitoring system based on his/her physical activities. This system avoids the use of individual sensor nodes, as they require separate processors, communication radios, and power systems, which eventually proves cumbersome for the soldier and may affect his/her free movement. The use of commonly available smartphone-based platforms 


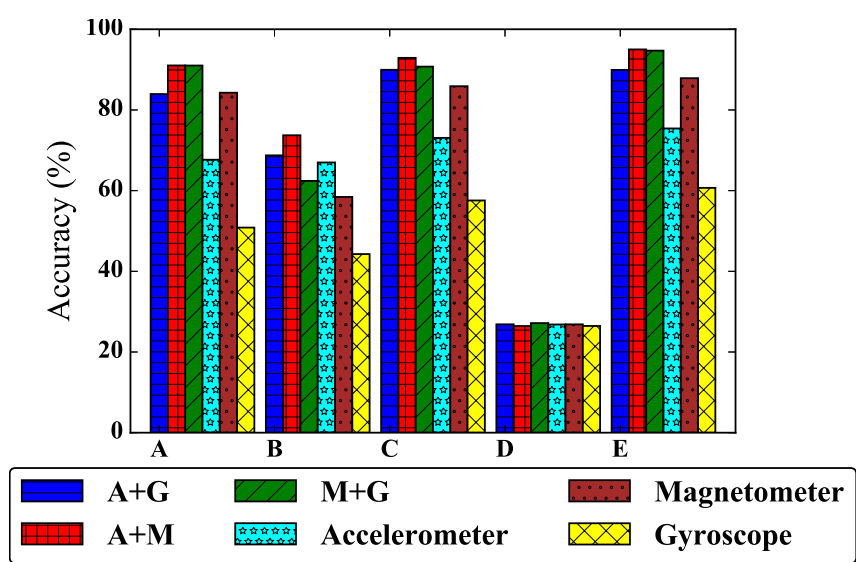

Fig. 4. Performance of classifiers in detecting activities with respect to various smartphone sensor combinations.

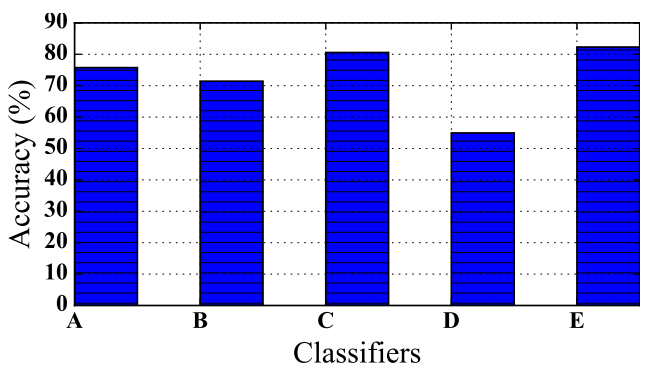

Fig. 5. Performance of classifiers in detecting activities with respect to the selected smartphone position on the human body and the chosen sensor combination.

for sensing, activity recognition, localization and communication tasks associated with a soldier not only makes this system more compact, but also cost-efficient and easily implementable without the need for extra modifications to a soldier's gear. The remotely-situated commander at the command center gets a comprehensive update of the group's activities as well as individual soldier's status with respect to the group. This system can be easily integrated with various fields such as health-care monitoring for elderly persons and with smart-homes for generating user context in a smarthome environment.

In the future, we plan on generalizing this model and train this system for identifying more activities as well as, improving the classification accuracy for the present activity sets. Additionally, we plan on integrating various bio-sensors to this platform, so as to make it a complete activity and health monitor.

\section{REFERENCES}

[1] F. Soldovieri and G. Gennarelli, "Exploitation of Ubiquitous Wi-Fi Devices as Building Blocks for Improvised Motion Detection Systems," Sensors, vol. 16, no. 3, p. 307, 2016.

[2] Y. Li, Y. Zhuang, H. Lan, Q. Zhou, X. Niu, and N. El-Sheimy, "A Hybrid WiFi/Magnetic Matching/PDR Approach for Indoor Navigation With Smartphone Sensors," IEEE Communications Letters, vol. 20, no. 1, pp. 169-172, Jan 2016.
[3] J. Lloret, A. Canovas, S. Sendra, and L. Parra, "A smart communication architecture for ambient assisted living," IEEE Communications Magazine, vol. 53, no. 1, pp. 26-33, January 2015.

[4] M. A. Hossain, J. Parra, S. M. M. Rahman, A. Alamri, S. Ullah, and H. T. Mouftah, "From sensing to alerting: a pathway of RESTstful messaging in ambient assisted living," IEEE Wireless Communications, vol. 23, no. 2, pp. 102-110, 2016.

[5] R. J. Lemmens, H. A. Seelen, A. A. Timmermans, M. L. Schnackers, A. Eerden, R. J. Smeets, and Y. J. Janssen-Potten, "To What Extent Can Arm-Hand Skill Performance of Both Healthy Adults and Children be Recorded Reliably Using Multiple Bodily Worn Sensor Devices?” IEEE Transactions on Neural Systems and Rehabilitation Engineering, vol. 23, no. 4, pp. 581-590, 2015.

[6] A. Anjum and M. U. Ilyas, "Activity recognition using smartphone sensors," in IEEE Consumer Communications and Networking Conference (CCNC). IEEE, 2013, pp. 914-919.

[7] A. Wang, G. Chen, J. Yang, S. Zhao, and C. Y. Chang, "A comparative study on human activity recognition using inertial sensors in a smartphone," IEEE Sensors fournal, vol. 16, no. 11, pp. 4566-4578, 2016.

[8] Y. Chen and C. Shen, "Performance analysis of smartphone-sensor behavior for human activity recognition," IEEE Access, vol. 5, pp. 30953110, 2017.

[9] San-Segundo, Echeverry-Correa, Salamea, and Pardo, "Human activity monitoring based on hidden markov models using a smartphone," IEEE Instrumentation Measurement Magazine, vol. 19, no. 6, pp. 27-31, 2016.

[10] M. T. Uddin, M. M. Billah, and M. F. Hossain, "Random forests based recognition of human activities and postural transitions on smartphone," in $20165^{\text {th }}$ International Conference on Informatics, Electronics and Vision (ICIEV), 2016, pp. 250-255.

[11] G. M. Weiss, J. W. Lockhart, T. T. Pulickal, P. T. McHugh, I. H. Ronan, and J. L. Timko, "Actitracker: A smartphone-based activity recognition system for improving health and well-being," in 2016 IEEE International Conference on Data Science and Advanced Analytics (DSAA), Oct 2016, pp. 682-688.

[12] Y. Lee and M. Song, "Using a smartwatch to detect stereotyped movements in children with developmental disabilities," IEEE Access, vol. 5, pp. 5506-5514, March 2017.

[13] A. GrÃijnerbl, A. Muaremi, V. Osmani, G. Bahle, S. Ãühler, G. TrÃüster, O. Mayora, C. Haring, and P. Lukowicz, "Smartphone-based recognition of states and state changes in bipolar disorder patients," IEEE fournal of Biomedical and Health Informatics, vol. 19, no. 1, pp. 140-148, Jan 2015 .

[14] N. Cardoso, J. Madureira, and N. Pereira, "Smartphone-based transport mode detection for elderly care," in 2016 IEEE $18^{\text {th }}$ International Conference on e-Health Networking, Applications and Services (Healthcom), Sept 2016, pp. 1-6.

[15] O. Baños, M. Damas, H. Pomares, I. Rojas, M. A. Tóth, and O. Amft, "A benchmark dataset to evaluate sensor displacement in activity recognition," in Proceedings of the ACM Conference on Ubiquitous Computing. ACM, 2012, pp. 1026-1035.

[16] REALDISP:Activity Recognition Dataset. UCI Machine Learning Repository. 